\title{
Conhecimento das gestantes de uma cidade do Norte de Minas sobre os tipos e os fatores que as influenciam na escolha da via de parto
}

\author{
Knowledge of pregnant women in a city of Northern Minas Gerais about the types and \\ factors that influence them in choosing the mode of delivery. \\ Conocimiento de las mujeres embarazadas en una ciudad del norte de Minas Gerais \\ sobre los tipos y factores que influyen en ellas para elegir el modo de parto
}

Mayara Pereira $^{1 *}$, Kelly Cristiane de Carvalho Aragão Sousa ${ }^{1}$, Ana Luiza Barbosa de Souza² Bruna Matos Gusmão², Kaick Alves Carvalho Laube ${ }^{2}$, Maria Clara Araújo Guedes², Caio Augusto Dias Gomes $^{2}$, Jaciara Neves Sousa ${ }^{2}$, Daniela Fernanda de Freitas ${ }^{2}$, Talita Antunes Guimarães ${ }^{1}$, Juliana Najara Alcântara Ferraz ${ }^{1}$.

\section{RESUMO}

Objetivo: Identificar o grau de conhecimento das gestantes em um munícipio do estado de Minas Gerais (MG) quanto aos tipos e os fatores que as influenciam na escolha da via de parto. Métodos: Este estudo tem uma abordagem descritiva, exploratória de natureza quanti-qualitativa, realizado a partir de entrevista semiestruturadas em encontros na unidade básica de saúde. Participaram da pesquisa 39 gestantes assíduas às consultas de pré-natal, que concordaram em assinar o termo de consentimento de livre esclarecido. Resultados: Por meio da pesquisa, concluímos que as gestantes participantes apresentam algum tipo de conhecimento acerca dos tipos de parto e que fatores como: recuperação pós-parto, o medo da dor e de surgirem complicações durante o parto, crises hipertensivas, desejo de realizar esterilização cirúrgica, opiniões de familiares e profissionais da saúde, são os principais responsáveis por influenciarem a gestante na escolha da via de parto. Conclusão: Em síntese há uma parcialidade quanto a escolha da via de parto, algumas das gestantes levantam a questão de menor risco durante o parto normal, outras relataram uma preocupação quanto danos na pelve, períneo, uretra e ânus, está é uma preocupação recorrente por esta razão acreditamos que o parto cesáreo seja mais bem aceito pelas gestantes.

Palavras-chaves: Gestação, Parto, Parto Abdominal, Parto Normal.

\begin{abstract}
Objective: Identify the degree of knowledge of pregnant women in a municipality in the state of Minas Gerais (MG) about the types and factors that influence them in choosing the mode of delivery. Methods: This study has a descriptive, exploratory approach of quantitative and qualitative nature, conducted from semi-structured interviews in meetings at the basic health unit. Thirty-nine pregnant women attending prenatal consultations, who agreed to sign the informed consent form, participated in the research. Results: Through the research, we concluded that the participating pregnant women have some kind of knowledge about the types of delivery and that factors such as postpartum recovery, fear of pain and complications during delivery, hypertensive crises, desire to perform Surgical sterilization, opinions of family members and health professionals are mainly responsible for influencing the pregnant woman in choosing the mode of delivery. Conclusion: In summary the study showed a bias while choosing the mode of delivery, some of the pregnant women raise the issue of lower risk during normal delivery, others reported a concern about damage to the pelvis, perineum, urethra and anus, is a recurring concern for this reason, we believe that cesarean delivery is better accepted by pregnant women.
\end{abstract}

Keywords: Pregnancy, Childbirth, Abdominal Birth, Normal Birth.

\footnotetext{
${ }^{1}$ Faculdade Vale do Gorutuba (FAVAG). Nova Porteirinha - Minas Gerais. *E-mail: mayarajaiba@bol.com.br

2 Universidade Estadual de Montes Claros (UNIMONTES). Montes Claros - Minas Gerais.
}

SUBMETIDO EM: 9/2019

ACEITO EM: 10/2019

PUBLICADO EM: 11/2019

$\overline{\text { REAS/EJCH | Vol.Sup.35 | e1825 | DOI: https://doi.org/10.25248/reas.e1825.2019 Página } 1 \text { de } 9}$ 


\section{RESUMEN}

Objetivo: Identificar el grado de conocimiento de las mujeres embarazadas en un municipio en el estado de Minas Gerais (MG) sobre los tipos y factores que influyen en ellas al elegir el modo de parto. Métodos: Este estudio tiene un enfoque descriptivo, exploratorio de naturaleza cuantitativa y cualitativa, realizado a partir de entrevistas semiestructuradas en reuniones en la unidad básica de salud. Treinta y nueve mujeres embarazadas que asistieron a consultas prenatales, que aceptaron firmar el formulario de consentimiento informado, participaron en la investigación. Resultados: a través de la investigación, concluimos que las mujeres embarazadas participantes tienen algún tipo de conocimiento sobre los tipos de parto y que factores como la recuperación posparto, el miedo al dolor y las complicaciones durante el parto, las crisis hipertensivas, el deseo de realizar La esterilización quirúrgica, las opiniones de los miembros de la familia y los profesionales de la salud son los principales responsables de influir en la mujer embarazada para elegir el modo de parto. Conclusión: En resumen, el estudio mostró un sesgo al elegir el modo de parto, algunas de las mujeres embarazadas plantean el problema de menor riesgo durante el parto normal, otras informaron una preocupación por el daño a la pelvis, el perineo, la uretra y el ano, es una preocupación recurrente para Por esta razón, creemos que las mujeres embarazadas aceptan mejor el parto por cesárea.

Palabras clave: Embarazo, parto, parto abdominal, parto normal.

\section{INTRODUÇÃO}

A gestação, desde sempre, atuou como um acontecimento de muito valor na vida das mulheres, sendo esse momento visto como sendo único, sem igual e marcante, notado pelas inúmeras alterações ocorridas na mulher diante da sua nova realidade de ser mãe. Diante disso, a gravidez, o parto e o puerpério constituem uma das experiências humanas mais significativas, proveitosas e valiosas para todas as pessoas que estão envolvidas neste processo (VELHO MB, et al., 2012).

O período que equivale à gestação, parto e puerpério é caracterizado como uma etapa na vida da mulher de extrema felicidade, incertezas e indagações, por isso, está necessita de um suporte familiar e profissional para que possa levar a diante a gestação e possa ter as informações necessárias para escolher a melhor forma de se ter e criar seu filho (RODRIGUES EM, 2011).

O parto caracteriza-se como um marco na vida das mulheres, que repercute bastante nas decisões que estas tomam devido às possíveis transformações físicas, emocionais e sociais que este pode acarretar (LOPES RCS, et al., 2005). Entre alguns motivos que influenciam a parturiente durante o trabalho de parto, podemos observar que a própria gravidez, as expectativas criadas pela gestante em relação ao parto e ao recém-nascido, a forma como essa foi informada durante o pré-natal bem como também uma gestação anterior podem interferir nas experiências vivenciadas pela mulher durante o parto podendo causar reações benéficas ou não (CAUS ECM, et al., 2012).

O modelo brasileiro de assistência ao parto vem sendo determinado pelo aumento de interferência e intervenções que contribuem para o aumento da escolha das gestantes pelo parto Cesáreo. Os índices brasileiros de cesáreas correspondem à cerca de $52 \%$ dos partos, estando este número muito elevado ao limiar máximo preconizado pela Organização Mundial da Saúde (OMS) que é de 15\% (NASCIMENTO RRP, et al., 2015).

Além disso, um estudo realizado durante 11 anos no estado do Paraná permitiu identificar que o parto cesáreo tem superado os partos normais/vaginais independente se o parto é realizado no setor público ou privado. Já em relação ao parto normal/vaginal, o Brasil ocupa o $2^{\circ}$ lugar entre os países da América tendo uma taxa de $68,5 \%$ de partos normais (NASCIMENTO RRP, et al., 2015).

No que diz respeito, a compreensão e aos fatores que interferem na escolha da via de parto, não é bem descrito se estes fatores são responsáveis ou não pelo aumento nas taxas de partos cesáreas. Sequer se tem conhecimento se há influência na escolha da via de parto, e por parte de onde surge esta influência. É provável que fatores não-clínicos, como nível socioeconômico, informação inadequada e conveniência dos profissionais de saúde, sejam responsáveis por influenciarem nesta escolha. 
Assim, o presente estudo tem como objetivo identificar o grau de conhecimento das gestantes em um munícipio do estado de Minas Gerais (MG) quanto aos tipos e os fatores que as influenciam na escolha da via de parto.

\section{MÉTODOS}

Trata-se de um estudo descritivo, exploratório de natureza quanti-qualitativa. O estudo foi desenvolvido em duas etapas, onde na primeira realizou-se a análise de documentos referentes ao grau de conhecimento das gestantes sobre os tipos e os fatores que as influenciam na escolha da via de parto disponíveis em bases de dados online do Google Acadêmico, BVS (Biblioteca Virtual em Saúde) e Scielo (Scientific Electronic Library Online) e em Revistas Especializadas na área da enfermagem, compreendidos no período de 2007 a 2017. A segunda etapa constitui-se na realização de entrevistas semi-estruturadas com o público alvo da pesquisa (gestantes).

O referido estudo foi realizado na o referido estudo foi realizado em uma Unidade Básica de Saúde (UBS), sendo esta, vinculada à Secretaria Municipal de Saúde pertencente a um munícipio do estado de Minas Gerais (MG), onde atualmente funcionam 4 equipes de ESF; as equipes 14, 17, 20, 23.

Foram incluídas as gestantes que frequentavam a unidade selecionada, assíduas às consultas de prénatal, independente do estado civil, religião, raça e idade gestacional, e que após o convite para participarem da pesquisa, concordassem em assinar o Termo de Consentimento Livre e Esclarecido, contendo todas as informações importantes da pesquisa como a metodologia e os objetivos da pesquisa. Foram excluídas as gestantes com menos de 18 anos.

Considerando que a unidade onde a pesquisa foi realizada possuíam 85 regulamente cadastradas no programa de pré-natal, apenas, 39 gestantes foram incluídas na pesquisa. Visto que, as demais gestantes não se propuseram a responder ao questionário.

A coleta de dados somente ocorreu após o recebimento do parecer favorável do Comitê de Ética em Pesquisa com Seres Humanos da universidade Estadual de Montes Claros sob no 2.398.841. Esse estudo foi realizado mediante entrevista semi-estruturada com a utilização de um questionário contendo questões abertas e fechadas totalizando 18 questões. Aplicação deste questionário ocorreu em um ambiente, onde a privacidade da gestante foi respeitada. Para garantir o anonimato das entrevistadas, substituíram-se os nomes das gestantes por iniciais. Porém, apesar de que se possa ter substituído os nomes por iniciais e a realização da pesquisa ser feita individualmente esta pode ter gerado constrangimento as entrevistadas.

Os dados obtidos por meio das questões fechadas do questionário aplicadas as gestantes foram digitadas numa planilha criada pela autora no programa do excel 2016, sendo estas, tabuladas de modo a permitir a caracterização das entrevistadas. Já as questões abertas, foram digitadas no programa do word 2016 de modo a permitir a visualização das respostas obtidas por meio da pesquisa com as gestantes.

\section{RESULTADOS E DISCUSSÃO}

A média de idades das gestantes entrevistadas variou entre $18 \pm 44$ anos sendo a predominância de faixa etária entre $18 \pm 28$ anos, correspondendo a $61,53 \%$ das entrevistadas. Desta forma percebe-se que as gestantes participantes se enquadram no que Caetano LC et al. (2011), assinalam como sendo e a idade ideal para ter filhos.

Estes descrevem que entre 20 e 29 anos, seja a idade ideal para a gravidez, visto que, os extremos da idade estão sempre ligados a complicações durante a gestação e trabalho de parto (CAETANO LC, et al., 2011).

Os dados a seguir, referem-se aos resultados obtidos mediante a aplicação de um questionário onde a pretensão foi descobrir o grau de conhecimento das gestantes de um munícipio do estado de Minas Gerais (MG) cadastradas na Unidade Básica de Saúde quanto aos tipos e os fatores que as influenciam na escolha da via de parto.

REAS/EJCH | Vol.Sup.35 | e1825 | DOI: https://doi.org/10.25248/reas.e1825.2019 Página $\mathbf{3}$ de $\mathbf{9}$ 
Quanto ao estado civil das gestantes, observou-se que $49 \%$ das entrevistadas são casadas, porém a união estável e o número de gestantes solteiras tiveram resultados próximos a $23 \%$ e $26 \%$ respectivamente. Um resultado similar a este, também foi encontrado por Dias WSL (2011), onde a predominância de gestantes entrevistadas era em sua maioria casadas e correspondia a $46,29 \%$, união estável e gestantes solteira correspondendo respectivamente a $29,62 \%$ e $24,07 \%$. Demonstrando assim, o perfil conjugal das gestantes entrevistadas (DIAS WSL, 2011).

Em relação ao grau de escolaridade das gestantes, foi possível observar que, 18 das entrevistadas concluíram o ensino médio, 02 não concluíram, 08 cursaram o nível superior, 6 estão cursando, 01 é pósgraduada, 02 decidiram não informar o seu grau de escolaridade e apenas 01 gestante possui o ensino fundamental incompleto.

Por meio desta pesquisa, e de pesquisas realizadas nos bancos de dados do IBGE (Instituto Brasileira de Geografia e Estatística), percebeu-se que a taxa de analfabetismo de pessoas na faixa etária $\geq 15$ anos diminuiu significativamente entre os anos de 1940/2010 passando de $56,0 \%$ para $9,6 \%$ tendo uma redução de $46,4 \%$ na taxa de analfabetismo. Além disso, o percentual de pessoas com pelo menos o ensino médio concluído é maior ou igual na população de 25 anos de idade, nas grandes regiões entre os anos de 2000/2010 evidenciou-se que a região sudeste apresentou crescimento no índice de pessoas que concluíram o ensino médio, passando de 27,0\% em 2000 para 40,1\% em 2010 havendo um acréscimo de 13,1\% nesta taxa (IBGE, 2010).

Quando questionadas quanto a sua renda familiar, 13 gestantes relataram ter apenas 01 salário mínimo, 14 informaram possuir 02 salários, 08 recebem 03 ou mais salários mínimos e apenas 04 gestantes não se propuseram a informar sua renda familiar. Esses dados permitem observar que, a maioria das gestantes $(46,15 \%)$ sobrevivem com uma renda mensal de $R \$ 1.908,00$ contradizendo os resultados de Peixoto CR et al. (2012), que observou, que $90,9 \%$ (70 gestantes) de suas entrevistadas (77 entrevistadas no total) apresentam renda familiar de até um salário mínimo o que corresponde a $\mathrm{R} \$ 954,00$. Além disso, de acordo com estudos realizados pelo IBGE em 2017, o rendimento mensal da população brasileira neste mesmo ano, equivale a um total de $R \$ 1.224,00$ o que representa pouco mais de 01 salário mínimo (PEIXOTO CR, et al., 2012).

Quando questionadas quanto ao planejamento da gestação, 22 responderam que a gravidez não fora planejada enquanto, 17 disseram ter planejada a gestação, identificando que $56,41 \%$ das gestantes entrevistadas não pretendiam terem filhos naquele dado momento.

Em um estudo realizado por Coelho EAC et al. (2012), 66,5\% das gestantes entrevistadas não planejaram a gestação, mostrando assim, que o número de gestação deste tipo ainda prevalece entre as mulheres brasileiras (COELHO EAC, et al., 2012).

Quando questionadas se eram primigestas ou multigesta, 21 gestantes disseram que era sua primeira gestação e 18 disseram que não era sua primeira gravidez. Destas 18 gestantes, 09 optaram pelo parto normal/vaginal e 09 decidiram pelo parto cesáreo. Abaixo, seguem as respostadas dadas por algumas das entrevistadas que explicaram o motivo de terem escolhido tais vias de parto:

\section{"O parto normal tem melhor recuperação". I.P}

"Escolhi o parto vaginal/normal porque é o melhor pra mim e para o nenê, muitos benefícios". L.S.O.

"Fiz o parto cesáreo pois tive pré-eclâmpsia". C.B.O.

"Meu bebê ficou atravessado e não nascia normal". L.N.A.

Conforme estudo realizado por Velho MB et al. (2014), um fator determinante na escolha da via de parto é a recuperação no pós-parto (VELHO MB, et al., 2014). Desta forma, muitas gestantes optam pelo parto normal, visto que, este possui uma recuperação mais rápida, permite a mulher realizar seus cuidados pessoais e com o bebê e as atividades referente ao lar. Em contrapartida, Novo JLVG et al. (2017), demonstra 
que em alguns casos existe necessidade da realização do parto cesáreo, como no caso da apresentação não cefálica do bebê e as síndromes hipertensivas na gestante sendo responsáveis pelo aumento das indicações de cesáreas nas instituições de saúde (NOVO JLVG, et al., 2017).

Quando investigadas quanto ao tipo de parto escolhido para a gestação atual, 24 gestantes responderam que preferem o parto normal/vaginal, 11 cesárea, 03 não decidiram e 01 não respondeu à pergunta. Ao inferirmos os motivos que as levaram a escolher estas vias de parto, as que optaram pelo parto normal relataram escolhe-lo devido: recuperação ser mais rápida, facilita o cuidado com o bebê, risco de infecções ser menor e não sentirem dor no pós-parto, sendo algumas destas respostas descritas abaixo:

"Pra poder cuidar do filho melhor". K.G.A.D.C.

"Escolhi esse parto por conta de a recuperação ser mais rápida". L.A.G.R.

"Escolhi o parto normal devido a recuperação ser mais rápida, melhor disponibilidade para cuidar do bebê, não corre o risco de ter uma infecção, não sente dores pós-parto etc". J.C.M.S.

Segundo estudos realizados por COSTA SP et al. (2014), grande número das entrevistadas escolhem o parto normal pois, este permite que as atividades diárias sejam realizadas normalmente após o parto, não existe os desconfortos gerados pelo parto cesáreo (dor pós-parto, cuidados com cicatriz cirúrgica), além de contribuir para uma recuperação mais rápida.

Quanto a escolha do parto cesáreo os principais motivos para a escolha foram: por causa da dor, fazer uso de medicamentos, problemas em controlar a pressão arterial, ter escolhido esta via na gestação anterior, por ser um parto rápido e pelo desejo de realizar a esterilização cirúrgica (laqueadura) estando algumas destas falas descritas a seguir:

"Escolhi o parto cesárea por ser mais rápido e por não ocasionar tanta dor". T.C.S.O.

"Escolhi o parto cesáreo pelo fato de já ter ocorrido desde a $1^{\circ}$ gravidez a cesariana, penso que a segunda gravidez também deve ser necessária". C.B.O.

"Escolhi o parto cesáreo por causa da dor e tomo remédios de controle e não sei qual seria a minha reação durante o parto". M.C.

"Escolhi o parto cesáreo porque a minha pressão está alta, estou com a vista embaraçada etc... e prefiro cesárea por causa da dor". I.R.D.S.

"Escolhi o parto cesáreo porque quero fazer laqueadura". A.G.

As crises hipertensivas, o desejo de realizar esterilização cirúrgica e medo da dor do parto, são descritos como os principais responsáveis por influenciarem a gestante a escolher o parto cesáreo segundo estudos realizados por vários autores (PIMENTEL TA e FILHO ECO, 2016; MINUZZI A e REZENDE CL, 2013).

Diante desse contexto, um levantamento realizado no banco de dados do Datasus demonstra que apenas no ano de 2015 forram efetuados cerca de 469 partos cesáreos, enquanto que a incidência de partos normais/vaginais no mesmo município e ano foram de 598 partos (BRASIL, 2018).

Segundo a Portaria № 306, de 28 de março de 2016 do Ministério da Saúde, a taxa de cesarianas recomendada para a população brasileira equivale a $25 \%-30 \%$. Isso demonstra que existe um aumento dessa taxa no município em estudo, visto que dos 1067 partos realizados no município 469 foram cesáreos o que representa $46,48 \%$ no número de partos realizados (BRASIL, 2016).

O perfil socioeconômico das gestantes não influencia a escolha da via de parto cesárea, contudo, a maioria das gestantes optam pela via de parto normal. Estes dados contrapõem o estudo realizado por Sgarbi AKG et al. (2013), que afirma quanto maior o nível de escolaridade maiores são as chances de ocorrência de via de parto cirúrgico. Algumas gestantes disseram se sentirem influenciadas por médico, outras gestantes, familiares, amigos e enfermeiro respectivamente, nesse contexto estudos ressaltam que muitas gestantes 
levam em consideração estas opiniões ao escolherem sua via de parto, sendo na maior parte influenciadas por profissionais e familiares (FIGUEIREDO NSV, et al., 2010; COSTA SP, et al., 2014).

Quanto ao conhecimento das gestantes sobre o parto normal, obtivemos as seguintes respostas:

"O parto normal é melhor para a mãe e para o bebê, por ser uma recuperação rápida e assim poder cuidar mais do bebê". L.A.G.R.

"Sei que é um parto natural onde não se aplica anestesia". E.C.S.S.

"Sei que o parto normal a dor é só até o nascimento. E o pós-parto e bem mais tranquilo e a recuperação mais rápida". R.S.S.S.

"Sei que é o melhor método, porque tem menos riscos pra gestante receberá alta do hospital mais rápido e a recuperação e mais rápida". M.C.

Em relação às vantagens do parto normal apontadas pelas gestantes, foi possível percebermos que a principal vantagem é a recuperação ser mais rápida, seguida de: não existir dor no pós-parto e cicatriz, menor risco para o binômio mãe/filho, menor chance de complicações e infecções no pós-parto, aumento do vínculo mãe/filho e por favorecer a amamentação. Estas respostas, podem ser visualizadas abaixo:

"A recuperação é rápida, não há dor pós-parto, alta do hospital é mais rápida, não há cicatriz, menor risco, uso de menos quantidade de medicação no pós-parto". S.N.N.S.N.

"Recuperação rápida, maior disponibilidade pós-parto para cuidados com o recémnascido". A.C.S.

"A mulher perde peso mais rápido, as dores do parto acabam no nascimento do bebê". M.C.S.

"A recuperação é rápida, favorece a amamentação, não há dor após o parto, alta do hospital mais rápida, risco de infecção é menor". J.C.M.S.

Segundo Cruz AP (2010), com o parto normal, a mulher possui menor risco de infecções, inexistência de ferida operatória, apresenta descida mais rápida do leite o que aumenta o vínculo mãe e filho, além de possibilitar uma recuperação mais rápida da mulher, que contribui para que esta realize sozinha os cuidados pessoais e com o bebê.

Quanto às desvantagens do parto normal nos deparamos com as seguintes respostas:

"Possíveis danos na pelve, possíveis danos no períneo, uretra e ânus, incontinência e fecal, dor no períneo". S.N.N.S.N.

"A dor e o medo de passar da hora da criança nascer". A.C.D.

"Possíveis danos a pelve, danos ao períneo uretra e ânus". T.C.S.O.

"Dizem que as contrações são bastantes doloridas". L.N.A.

Conforme estudos feitos por Melo JKF et al. (2015), as principais desvantagens do parto normal relatadas pelas gestantes são: as intensas contrações uterinas sofridas durante o trabalho de parto, as dores decorrentes das contrações uterinas e o incômodo gerado na mulher quando é submetida a episiotomia.

Assim outros autores acrescentam dizendo que, um pequeno número de mulheres permanecerá com o períneo íntegro depois o parto normal. Isso acontece, pois, a grande maioria das gestantes estão sujeitas a episiotomia ou sofrerão algum tipo de laceração perineal durante o parto (COSTA ADSCD, et al., 2014).

Mediante ao conhecimento das gestantes sobre o parto cesáreo, obtivemos as seguintes respostas:

"O parto cesáreo as vezes é sugerido pelo médico pela saúde da gestante porque a gestante pode ter problema na gestação". L.A.G.R. 
"É um processo cirúrgico, consiste em um corte na parede abdominal e no útero, deixando a recuperação mais lenta". S.N.N.S.N.

"É um parto sem dor, pois é aplicado a anestesia e a recuperação é mais prolongada". B.G.

"Na cesárea não se sente dor, por conta da anestesia porém pode sofrer mais no pós-operatório com as dores da cicatriz". R.S.S.S.

Já no que diz respeito as vantagens do parto cesáreo, observamos que as gestantes acham este o melhor, pois: ela não sente dor durante o parto, o parto é mais rápido que o normal, tem a opção de agendar o dia do parto e escolher o médico que realizará o mesmo; algumas destas podem ser visualizadas abaixo:

"Apenas a opção de poder agendar o dia do parto e não sentir as dores do parto (em alguns casos)". D.C.O.

"Menos dor e sofrimento". A.C.D.

"O nascimento é mais rápido, a mulher não sente dores durante o processo devido a anestesia". T.C.S.O.

"Mais rápido, marca-se dia e a hora, escolhe-se o médico que você quer que acompanhe, diferente do parto normal que é o que tiver no dia de plantão no hospital". C.B.O.

Por meio de pesquisas realizadas por Gama ADS et al. (2009), o parto cesáreo apresenta as seguintes vantagens: inexistência do processo doloroso durante o trabalho de parto, possibilidade de agendamento do parto, e da possibilidade de se escolher o médico que realizará o parto, corroborando com estudos da literatura (PIRES D, et al., 2010).

A via de parto possibilita a realização da esterilização cirúrgica (laqueadura) caso este seja o desejo da mulher (COPELLI FHS, et al., 2015). As gestantes que participaram da atual pesquisa relataram as mesmas vantagens descritas pelos autores mencionados acima.

Quanto às desvantagens desse tipo de parto, deparamos com as seguintes respostas: que a recuperação materna é mais lenta, ocorre o atraso da lactação, o cuidado com o bebê torna-se mais difícil, o risco de infecções e complicações são maiores além de que o bebê não esteja preparado para nascer. Abaixo estão descritas algumas das respostas dadas pelas gestantes:

"Recuperação mais lenta, atraso na lactação, dobro na permanência hospitalar, dores e dificuldades na localização dos pontos". S.N.N.S.N.

"Cuidar do bebê é mais difícil". A.D.A.

"Recuperação lenta, dor para se locomover, atraso na lactação, risco de infecção inflamação etc, cicatriz". J.C.M.S.

"Pós-parto mais complicado, maior risco de infecção, amamentação tardia, o bebê não está preparado para nascer". L.S.O.

Estas respostas acimas citadas, também foram encontradas por diversos autores em alguns estudos realizados com gestantes e puérperas quanto as desvantagens do parto cesáreo, sendo algumas delas: maior risco de infecções e complicações (hemorragias), recuperação pós-parto tardia, maiores chances do recémnascido desenvolver problemas respiratórios, risco da puérpera desenvolver trombose venosa profunda, além disso, a descida do leite materno é mais lenta quando o parto realizado é o cesáreo (NASCIMENTO RRP, et al., 2015; MELO JKF, et al., 2015; CRUZ AP, 2010; GUEDES GW, et al., 2016)

Perguntou-se as gestantes como elas tiveram acesso às informações sobre os tipos de parto e as vantagens e desvantagens de cada um. Das 39 entrevistadas, $27 \%$ disseram ter recebido tais informações por meio de familiares e amigos, $17 \%$ por meio da internet, $18 \%$ adquiriram as informações por meio de suas 
experiências, $14 \%$ com outras gestantes, $8 \%$ por meio de leitura em revistas, livros e jornais, $6 \%$ por meio do médico, $6 \%$ por meio do enfermeiro e apenas $4 \%$ por meio de programas de televisão.

Em estudo realizado Junior TL, et al. (2013), demonstra que as gestantes participantes da pesquisa, também adquirem informações acerca das vias de parto, por meio da televisão, durante o pré-natal (com profissionais da saúde), amigos, vizinhos e por meio de experiências adquiridas de partos anteriores, entrando assim, em concordância com os dados coletados pelas autoras.

Percebemos que as gestantes entrevistadas apresentam algumas dúvidas quanto aos tipos de parto, sendo que 21 gestantes relataram estar nesta situação. Identificou-se também, que as consultas de pré-natal realizadas na unidade de saúde propiciam as gestantes bastante informações pertinentes aos tipos de parto, visto que, 23 participantes relataram que recebem informações do médico ou enfermeiro quanto as vias de parto. Isso é possível pois, durante as consultas de pré-natal os profissionais da saúde oferecem as gestantes orientações quanto a gestação, cuidados com elas e seu bebê, quanto ao trabalho de parto entre outras informações que preocupam as gestantes. Esta troca de informações contribui para que a gestação, o trabalho de parto e parto ocorram com o mínimo de complicações possíveis (QUEIROZ MVO, et al., 2016).

\section{CONCLUSÃO}

Os resultados apontam a complexidade que envolve o processo de escolha da via de parto, influenciada por fatores como pré-eclâmpsia durante a gestação, há ainda àquelas que desejam realizar a laqueadura. Contudo nesta população ressaltamos uma parcialidade enquanto a escolha da via de parto, algumas das gestantes levantam a questão de menor risco durante o parto normal, outras relataram uma preocupação quanto danos na pelve, períneo, uretra e ânus, está é uma preocupação recorrente por esta razão acreditamos que o parto cesáreo seja mais bem aceito pelas gestantes. $O$ pequeno número de gestantes entrevistadas é uma limitação do estudo o que pode ter interferido nos resultados encontrados, contudo não possibilitando generalizações.

\section{REFERÊNCIAS}

1. BRASIL. Ministério da Saúde. Gabinete do Ministro. Portaria ํㅜ 306, de 28 de março de 2016. Aprova as Diretrizes de Atenção à Gestante: a operação cesariana. Diário Oficial União. Brasília, DF, 29 mar 2016. Seção: 1. p. 58

2. BRASIL. DATASUS. 2018. Tecnologia da Informação a Serviço do SUS. Disponível em: http://tabnet.datasus.gov.br/cgi/tabcgi.exe?sinasc/cnv/nvMG.def. Acesso em: 09 abr. 2018.

3. CAUS ECM, et al. O processo de parir assistido pela enfermeira obstétrica no contexto hospitalar: significados para as parturientes. 2012.

4. CAETANO LC, et al. Gravidez depois dos 35 anos: uma revisão sistemática da literatura. Revista mineira de enfermagem, 2011; 15: 579-587.

5. COELHO EAC, et al. Associação entre gravidez não planejada e o contexto socioeconômico de mulheres em área da Estratégia Saúde da Família. Acta Paulista de Enfermagem, 2012; 25: 415-422.

6. COSTA SP, et al. Parto normal ou cesariana? Fatores que influenciam na escolha da gestante. Revista de Enfermagem da UFSM, 2014; 4: 1-9, 2014.

7. COPELLI FHS, et al. Fatores determinantes para a preferência da mulher pela cesariana. Texto Contexto Enferm, 2015; 24: 336-43, 2015.

8. CRUZ AP. Parto Natural. Conselho Regional de Enfermagem. São Paulo. 2010.

9. FIGUEIREDO, NATHÁLIA SV. Fatores culturais determinantes da escolha da via de parto por gestantes. HU Revista, $2010 ; 36$.

10. GUEDES GW, et al. Conhecimentos de gestantes quanto aos benefícios do parto normal na consulta pré-natal. Rev. enferm. UFPE online, 2016; 10: 3860-3867.

11. IBGE. Censo Demográfico 2010. Resultados gerais da amostra. 2012. Acesso em: 06/04/2018

12. JUNIOR TL, et al. Escolha da via de parto: expectativa de gestantes e obstetras. Revista Bioética, 2013; 21: 509517.

13. LOPES RCS, et al. O antes e o depois: expectativas e experiências de mães sobre o parto. Psicologia: reflexão e crítica. Porto Alegre. 2005; 18: 247-254. 
14. MINUZZI A, REZENDE CL. Fatores de influência na escolha da viade parto: uma revisão de literatura. Revista Uningá Review, 2013; 14: 11-11.

15. MELO JKF, et al. Vantagens e desvantagens do parto normal e cesariano: opinião de puérperas. Revista de Pesquisa Cuidado é Fundamental Online, 2015; 7: 3197-3205.

16. NOVO JLVG, et al. Indicações de partos cesáreos em hospitais de atendimento ao Sistema Único de Saúde: baixo e alto riscos. Revista da Faculdade de Ciências Médicas de Sorocaba, 2017; 19: 67-71.

17. NASCIMENTO RRP, et al. Escolha do tipo de parto: fatores relatados por puérperas. Revista Gaúcha de Enfermagem, 2015; 36: 119-126.

18. PEIXOTO CR, et al. Perfil das gestantes atendidas no serviço de pré-natal das unidades básicas de saúde de Fortaleza-CE. Revista Mineira de Enfermagem, 2012; 16: 171-177.

19. PIMENTEL TA, OLIVEIRA-FILHO EC. Fatores que influenciam na escolha da via de parto cirúrgica: uma revisão bibliográfica. Universitas: Ciências da Saúde, 2016; 14.

20. PIRES D, et al. A influência da assistência profissional em saúde na escolha do tipo de parto: um olhar sócio antropológico na saúde suplementar brasileira. Revista brasileira de saúde materno infantil= Brazilian journal of mother and child health. Recife. 2010; 10: 191-197.

21. QUEIROZ MVO, et al. Grupo de gestantes adolescentes: contribuições para o cuidado no pré-natal. Revista Gaúcha de Enfermagem, 2016; 37.

22. RODRIGUES EM, et al. Protocolo na assistência pré-natal: ações, facilidades e dificuldades dos enfermeiros da Estratégia de Saúde da Família. Revista da Escola de Enfermagem da USP, 2011; 45: 1041-1047.

23. SGARBI AKG, et al. Estudos comparativos sobre fatores que influenciam a Escolha do tipo de parto pelas gestantes. In: Inter. 2013; p. 72-81.

24. VELHO MB, et al. Vivência do parto normal ou cesáreo: revisão integrativa sobre a percepção de mulheres. Texto Contexto Enferm [Internet]. 2012 abr/jun; [cited 2016 Jul 4]; 21 (2): 458-66.

25. VELHO MB, et al. Parto normal e cesárea: representações sociais de mulheres que os vivenciaram. Revista Brasileira de Enfermagem, 2014; 67: 282-289. 